\title{
RECHTS- UND SACHMÄNGELHAFTUNG IM RÖMISCHEN KAUFRECHT: STIPULATIO SIMPLAE UND AUSSCHLUSS DER WANDELUNG ${ }^{1}$
}

\author{
RESPONSABILIDADE POR EVICÇÃO E VÍCIOS OCULTOS NO DIREITO ROMANO DA COMPRA E \\ VENDA: STIPULATIO SIMPLAE E EXCLUSÃO DA REDIBIÇÃO
}

LIABILITY FOR EVICTION AND LATENT DEFECTS IN THE ROMAN LAW OF SALE: STIPULATIO

SIMPLAE AND EXCLUSION OF RESCISSION

André Nunes Conti*

\begin{abstract}
Zusammenfassung:
Die noch heute relevante Unterscheidung zwischen Rechts- und Sachmängelhaftung war bereits im klassischen römischen Privatrecht bekannt. Diese zwei Arten der Mängelhaftung hatten schon damals unabhängige Grundlagen und folgten je einer eigenen Logik. Einerseits ergab sich die Rechtsmängelhaftung meistens aus einer genau zu diesem Zweck abgeschlossenen Stipulation, wogegen die Sachmängelhaftung hauptsächlich im Ädilenedikt zur Marktregulierung vorgesehen war, und zwar in Form der ädilizischen Klagen actio redhibitoria und quanti minoris. Beide Haftungsformen durchkreuzen sich anscheinend im interessanten Fragment D. 21, 1, 48, 8 des Pomponius, das entgegen der vorherrschenden Meinung eine Hypothese der Verbindung zwischen beiden Seiten der Mängelhaftung des Verkäufers enthält. Würde nämlich die Rechtsmängelhaftung vertraglich gemindert (d.h. würde die „stipulatio simplae“ anstelle der üblichen „,duplae“ abgeschlossen, worin die sogenannte ,venditio simplaria“ bestanden haben müsste), so würde das Schweigen der Parteien in Bezug auf Sachmängelhaftung als ihren Ausschluss angenommen. Diese Auslegung des Fragments stützt sich nicht nur auf eine grammatikalische Analyse des Textes (die sich auf das interessante, sonst nirgends auftauchende Wort ,simplaria“ fokussiert), sondern auch auf mehrere einschlägige Rechtsquellen, insbesondere auf den ägyptischen Papyrus P. Cair. Preis. 1, wo die von Pomponius erwähnte Vermutung anscheinend von einem Anwalt in einem realen Fall angewandt wird.
\end{abstract}

Stichwörter: Römisches Recht. Kaufvertrag. Mängelhaftung des Verkäufers. Rechtsmängelhaftung. Sachmängelhaftung. Venditio simplaria.

\footnotetext{
1 Das Thema wurde im Sommer 2018 im Rahmen eines Seminars an der LMU München erarbeitet. Herrn Prof. Platschek, dem ich die Fragestellung sowie die hauptsächlichen Grundlinien der Forschung schulde, will ich jetzt meinen aufrichtigen Dank und meine tiefe Wertschätzung ausdrücken. Außerdem verweise ich auf seinen Beitrag zum selben Thema in U. Babusiaux/M. Igimi, Messages from Antiquity (2019), „Strict Liability for defects as to Quality of an Object Sold".

* Estudante na Faculdade de Direito do Largo de São Francisco, com Zertifikatsstudium pela LudwigMaximilians-Universität München (LMU), e cursando Licence en Droit pela Université Lyon III.
} 
Resumo:

O Direito Romano clássico já conhecia a distinção ainda hoje relevante entre as duas principais modalidades de responsabilidade do vendedor: por evicção e por vícios ocultos. Sabe-se que elas tinham fundamentos diversos e seguiam cada uma sua lógica própria. Enquanto a responsabilidade por evicção decorria da celebração de uma stipulatio especificamente para esse fim, a responsabilidade por vícios ocultos estava prevista no Edito dos Edis Curuis para a regulação do mercado, materializando-se nas ações redhibitoria e quanti minoris. Contudo, esses dois planos parecem entrecruzar-se no curioso fragmento D. 21, 1, 48, 8 de Pompônio, o qual, contrariamente ao que ainda sugere a interpretação majoritária, aparentemente contém uma hipótese de interligação entre os dois âmbitos de responsabilidade do vendedor. Segundo ele, caso reduzida a responsabilidade por evicção (i.e. quando celebrada a stipulatio simplae no lugar da habitual duplae, configurando-se desse modo a assim chamada "venditio simplaria"), o silêncio das partes quanto à responsabilidade por vícios ocultos faria presumir sua exclusão. Essa leitura do fragmento encontra arrimo não apenas numa análise gramatical de seu texto (focalizada no interessante vocábulo "simplaria", palavra que não ocorre em absolutamente nenhum outro texto latino), mas também em diversas fontes jurídicas relacionadas com o tema, das quais se destaca o papiro egípcio P. Cair. Preis. 1, em que a presunção contida no fragmento de Pompônio parece ser usada como argumento de um advogado num caso real.

Palavras-chave: Direito Romano. Compra e venda. Responsabilidade do vendedor. Evicção. Vícios ocultos. Venditio simplaria.

\begin{abstract}
:
Classical Roman Law already knew the distinction between liability for eviction and latent defects, which had independent foundations and did not follow the same logic. The liability for eviction arose from the celebration of a stipulatio specifically for this purpose, whereas the liability for latent defects was foreseen in the Ediles Curules Edict for market regulation, in form of the actiones redhibitoria and quanti minoris. Notwithstanding, these two plans seem to intersect in the curious fragment D. $21,1,48,8$ of Pomponius, which, contrary to what suggests the prevailing opinion, contains a hypothesis of interconnection between the two sides of the vendor's liability. According to him, if the liability for eviction was reduced (i.e. when stipulatio simplae was celebrated instead of the usual duplae, wherein the so called "venditio simplaria" consisted), the silence of the parties regarding liability for latent defects would lead to a presumption of its exclusion. This reading of the fragment finds support not only in a grammatical analysis of its text (focused mainly on the interesting word "simplaria", that occurs in no other classical Latin document whatsoever), but also in several legal sources related to the subject, specially the Egyptian papyrus P. Cair. Preis. 1, where the presumption mentioned by Pomponius is apparently applied by a lawyer in a real case.
\end{abstract}

Keywords: Roman Law. Sale contract. Vendor's liability. Liability for eviction. Liability for latent defects. Venditio simplaria. 
I. Darstellung des Problems

Die kleine Digestenstelle D. 21, 1, 48, 8 (Pomp. 23 Sab.) sieht auf den ersten Blick so direkt, schlicht und klar aus, dass praktisch keine Auslegung bei ihr nötig zu sein scheint. Pomponius macht hier eine Anmerkung, die wenig mit den nach der Reihenfolge der Kompilatoren vorigen Fragmenten zu tun hat, und die sogar überflüssig oder selbstverständlich klingen kann. So schreibt Pomponius:

D. 21, 1, 48, 8 (Pomp. 23 Sab.): Simplariarum venditionum causa ne sit redhibitio, in usu est.

Es handelt sich um einen Ausschluss der Haftung für Sachmängel bei den sogenannten simplariae venditiones, was als Gewohnheit gekennzeichnet wird (in usu est). Obwohl die Stelle offensichtlich außer Kontext steht (LENEL, 1889, p. 134), ${ }^{2}$ weiß man, dass die Rede hier zweifellos von der Sachmängelhaftung beim Sklavenmarktkauf sein muss. Im Buch 23 ad Sabinum wird nämlich das Edikt der kurulischen Ädilen von Pomponius kommentiert (in den anderen Paragraphen von D. 21, 1, 48 geht es hauptsächlich um die Anwendbarkeit der ädilizischen Klage in verschiedenen Situationen) (Ibid.), und in D. 21, 1, 48, 8 wird die redhibitio, d.h. die Wandelung (ein ädilizisches Rechtsbehelf) erwähnt.

Als zentrales Problem taucht die Übersetzung des Hapaxlegomenons „simplaria“ auf. Da es die einzige Anwendung des Worts in der ganzen lateinischen Überlieferung ist, hat man keine sonstige Anwendung zum Vergleich mit der Stelle in Betrachtung, sodass die grammatikalische Bedeutung des Wortes ganz vom Sinn ebendieses Fragments abhängt, das heißt, von seinem juristischen Sinn. Hier wird ein Teil des Wortlauts der auszulegenden Stelle ausnahmsweise zum Ergebnis der juristischen Auslegung, statt Anfang derselben zu sein.

Natürlich ergeben sich einige Begrenzungen der Bedeutungsmöglichkeiten des Wortes ,simplaria“ schon daraus, dass man seine Verwandtschaft entweder mit simplex oder mit simpla erkennt: die Bedeutung und folglich die Übersetzung werden sicherlich mit „einfach“ zu tun haben. Die merkwürdige Umgestaltung des Wortes in der Stelle suggeriert dennoch eine absichtliche Distanzierung von der allgemeinen Bedeutung des Wortes „einfach“. Mithilfe einer Analyse der Struktur des lateinischen Wortes ,simplaria“ (simpl -ari -a) darf man mögliche Absichten Pomponius zur Anwendung dieser Form erahnen. Eine reine grammatikalische Diskussion wäre aber fruchtlos. Wie gesagt, es

2 „Compilatores in hac §o plures Pomponii sententias inepte videntur in breve coegisse“, was bezüglich der Fragmenten, die D. 21, 1, 48 pr.-8 entsprechen, gesagt wird. Es sind tatsächlich viele Anmerkungen Pomponius zur Sachmängelhaftung beim Marktkauf von Sklaven, die aber wenig miteinander zu tun haben. 
wird vor allem der juristische Sinn der ganzen Stelle dafür verantwortlich sein, dass ihre genaue Bedeutung ermittelt wird.

Dass es sich jedenfalls um keine eindeutige Stelle handelt, zeigen schon die zahlreichen Auslegungen, die bereits vorgeschlagen worden sind. Eigentlich gibt es drei Hauptmeinungen dazu:

\section{Vertragsmäßiger Ausschluss der Wandelung}

Zunächst gibt es diejenigen, die die simplaria venditio als Kauf mit ausdrücklichem, vertragsmäßigem Ausschluss der Haftung für Sachmängel verstehen. Der „einfache“ oder ,schlichte“ Kauf sei zustande gekommen, wenn die Parteien die Haftung für Sachmängel vertraglich absolut ausgeschlossen hätten, ${ }^{3}$ was normalerweise eine Reduktion des Preises als natürliche Folge verursacht habe. Diese ist zurzeit die herrschende Meinung, und wird z.B. sowohl von É. Jakab (1997, p. 183 et seq.) als auch von B. Kupisch (2002, p. 43) vertreten, die sonst grundsätzliche Zwistigkeiten haben. Sie ist in der englischen Übersetzung des Digests von A. Watson zu finden (was wahrscheinlich damit zu tun haben wird, dass eben sie im Oxford Latin Dictionary auftaucht ${ }^{4}$ ):

It is not our practice to allow rescission in the case of sales where undertakings have been specifically excluded (WATSON, 1985, p. 617). ${ }^{5}$

Vertreter dieser Meinung waren schon B. Windscheid (1906, p. 692), L. Mitteis (1912, p. 192) und E. Rabel (1955, p. 109), die damals als Mindermeinung galten. Auch L. Wenger (1953, p. 778), P. Meyer (1921, p. 258) und, im spezifischen Bereich der Papyrologie, F. Pringsheim (1950, p. 483 et seq.). ${ }^{6}$

3 Gemäß D. 2, 14, 31 Ulp. 1 ed. aed. cur.: Pacisci contra edictum aedilium omnimodo licet, sive in ipso negotio venditionis gerendo convenisset, sive postea.

4 „Venditio [simplari]a, a sale from which any warranty is excluded" (GLARE, 1945).

5 Und sie ist auch in der französischen Übersetzung Hulos zu erkennen, obgleich mit weiteren Behauptungen: On est dans l'usage de faire des ventes toutes simples, c'est-à-dire sans louer ni blâmer la chose, afin d'empêcher l'effet de la redhibition (HULOT, 1804, p. 183). Hulot geht davon aus, beim Schweigen sei die Haftung für Sachmängel ausgeschlossen worden, was aber weder für den Sklavenmarktkauf — bei dem solche Haftung ex lege galt, obwohl sie abbedungen werden konnte (vgl. D. 2, 14, 31 Ulp. 1 ed. aed. cur.) — noch bei normalen Käufen, bei denen es sowieso keine Sachmängelhaftung gab, die abbedungen werden konnte; andersherum musste die Haftung bei ihnen ausdrücklich übernommen werden (vgl. HONSELL, 1984, p. 56).

6 Er analysiert verschiedene Papyri, in denen es um Käufe ohne Garantie geht, und zieht besonders eindeutig den Schluss, es habe in den Quellen viele Synonymen für solche Käufe gegeben, zwischen denen eben die simplaria venditio bei Pomponius zu erwähnen sei. 
Weitere Beispiele sind W. Ernst (1999, p. 216), E. Weiß (1916, p. 172) ${ }^{7}$ und G. Impallomeni (1955, p. 21). ${ }^{8}$ Aber als herrschende Meinung hat sie natürlich noch etliche Vertreter. ${ }^{9}$

\section{Nicht-Erforderlichkeit der stipulatio duplae}

Eine andere Auslegung des Wortes simplaria, die von der heute herrschenden Meinung zu unterscheiden ist, taucht in einigen lateinischen Wörterbüchern auf:

„SIMPLARIUS, a, um, adj. simplaris, simplus, Simplaria venditio, pro qua necesse venditor non habet de dupla emptori repromittere*, sed de simpla dumtaxat. Pomp. Dig. 21, 1, 48“ (FORCELLINI, 1835). ${ }^{10}$

Die Wörterbücher, die so eine Definition für das Wort „simplaria“ anbieten, weisen auf keine Quelle hin, die ihre Auslegung rechtfertigt. Die auffallende Ähnlichkeit mit der Ausdrucksweise des Baldus in einer Anmerkung von ihm zum Kommentar des Accursius bezüglich der Stelle D. 21, 1, 48, 8 führt zur Vermutung, dass er ein möglicher Ursprung dieser Meinung sein könnte:

„Simplariae venditiones quae sint, ignorat hic Accursius. Sunt autem pro quibus necesse venditor non habet de dupla emptori repromittere $[\ldots]^{\text {“. }}{ }^{11}$

Hier geht er davon aus, es gäbe gemäß dem Ädilenedikt einen allgemeinen Garantiezwang, aufgrund dessen der Käufer immer den Abschluss der stipulatio duplae für den Fall der Eviktion verlangen durfte. ${ }^{12}$ Simplaria venditio wäre in diesem Sinn derjenige Kauf, bei dem (wegen des Gegenstandes) solche Stipulation nicht gefordert werden konnte, was — so lässt sich diese Meinung interpretieren — zur Folge hatte, dass

7 In dieser Möglichkeit des Ausschlusses will er sogar die „Verkörperung der vollen Vertragsfreiheit” sehen.

8 In der „dichiarazione generale di non assumere la garanzia per i defetti occulti“ sieht er ein pactum de non petendo - das natürlich nicht die spezifische Haftung für Arglist ausschließt.

9 Vgl. (HONSELL; MAYER-MALY; SELB, 1987, p. 317); (DONADIO, 2004, p. 258-259); (MEMMER, 1990, p. 16), dieser letzte mit ausführlichem Literaturhinweis.

10 Eine fast identische Definition findet sich bei K. E. Georges (1998, p. 2.074): „Simplarius, a, um (simplus), einfach, venditio $=u b i$ venditor non cogitur de dupla repromittere, sed de simpla tantum, Pompon. dig. 21, $1,48 \S 8$ ".

11 Baldus in Accursius, (1627, p. 1.972) (Unterstreichung hinzugefügt). Auch Guillaume Budé (1543, p. 350351) hat sie vertreten.

12 Die Theorie des Garantiezwangs beim Ädilenedikt wurde erst von É. Jakab (1997, p. 183; 2009). Auch dazu: (ERNST, 1999, p. 216). B. Kupisch hat versucht, noch eine weitere Auslegung der Stelle D. 21, 1, 28 (Gai.) und das durch sie erzeugte Fristenproblem aufzustellen, aber die durch É. Jakab an diesen Versuch geübte Kritik ist völlig überzeugend. Dieses Problem wird hier nicht erörtert, weil es natürlich eine unverhältnismäßige Ablenkung vom Thema wäre. Was aber dem prozessualen Mechanismus des Ädilenedikts betrifft, wird hier der durch É. Jakab aufgestellten Theorie gefolgt. 
auch die Sachmängelhaftung ausgeschlossen wurde, denn sie wurde meistens zusammen mit der Haftung für Rechtsmängel vereinbart, sodass die Unterlassung der stipulatio duplae auch zugleich eine Unterlassung der Vereinbarung der Sachmängelhaftung mit sich brachte.

Baldus spricht nicht von der stipulatio simplae, die den Wörterbüchern zufolge verlangt werden konnte. Sie wird ebenso wenig von H. Dernburg (1912, p. 765) erwähnt, der genau dieselbe Meinung Baldus vertritt. ${ }^{13}$

Es ist nicht klar, was für Quellen die Wörterbücher für diese Behauptung hatten, dass die stipulatio simplae bei der venditio simplaria erforderlich war. Das kann möglicherweise schlicht als Erklärung für das Wort „simplaria“ als Verwandte mit „simplex“ gewesen sein. Es ist auf jeden Fall eine sehr interessante Behauptung, die dem hier zu machenden Vorschlag zur Auslegung der Stelle einigermaßen ähnlich, aber doch nicht gleich sein wird.

\section{Kauf geringwertiger Sachen}

Eine dritte Auslegungsmöglichkeit nimmt das Wort simplaria als Nomen, oder will das Wort „rerum“ implizit in der Stelle lesen, damit die simplariarum venditionum causa eigentlich zu simplariarum , rerum “venditionum causa wird, das heißt, hier handle es sich um den Kauf von „simplariae res“, was als „geringwertige Sachen“ verstanden wird. Pomponius sage also, es sei eine Gewohnheit, dass die Wandelung bei den Käufen von geringwertigen Sachen keinen Platz greife. Diese Meinung taucht z.B. in der italienischen Übersetzung der Digesten von Schipani (2011, p. 83) auf:

„Non si usa effettuare redibizione a proposito di cose di tenue valore". 14

Diese Meinung hat, wie schon gesagt, H. Dernburg (1912, p. 765) vertreten, genauso wie Donellus (1861, p. 809), und Glück (1819, p. 45). Sie ist bereits die herrschende Meinung gewesen, auch wenn, genau gesehen, unbegründet (WINDSCHEID, 1906, p. 692). Sie ist häufig eine Spezifikation der Meinung der nicht-Erforderlichkeit der stipulatio duplae gewesen, indem die Käufe geringwertiger Sachen eben diejenigen seien,

13 Auch wenn mit der zusätzlichen Anmerkung, dass diese nicht Erforderlichkeit der stipulatio duplae z.B. bei den Käufen von geringwertigen Sachen zutrifft, weshalb er auch als Vertreter einer anderen Hauptmeinung zur Auslegung des Worts simplaria angesehen werden kann.

14 Ebenso bei G. Vignali (1857, p. 450): „L'uso porta, che non vi sia redibitoria per vendite minute“. Wahrscheinlich auch in der spanischen Übersetzung von D’Ors dieselbe Meinung vertreten wird: „Está en uso que no exista redhibición en razón de las ventas más corrientes“ (D’ORS, 1972, p. 56). Aber es ist nicht klar, ob er simpliciorum statt simplariarum gelesen hat, und so die venditiones simpliciores als ,,ventas mas corrientes" übersetzt hat, oder ob er die simplariarum venditiones tatsächlich als Käufe von geringwertigen Sachen verstanden hat, und gesagt, dass sie die häufigsten sind. 
bei denen die stipulatio duplae nicht erforderlich sei (wie es in D. 21, 2, 37, $1^{15}$ zu lesen ist).

Sie ist auf jeden Fall von der Meinung Accursius (1627, p. 1972) in der Magna Glosa zu unterscheiden. Da behauptet er, die simplariae res wären diejenigen, die keine Seele (anima) und keinen Leib (corpus) haben, also, die Rechte (quae in iure consistunt), wie das Erbe, oder auch die Sachen die keine Ernährung brauchen (carentes vitali alimento).

Soweit merkt man schon, dass die am Anfang eindeutig aussehende Stelle eigentlich überhaupt nicht so klar ist, wie man denken könnte. Und obwohl die herrschende Meinung des Kaufes mit ausdrücklichem Ausschluss der Wandlung heute praktisch nicht bestritten wird, sind doch einige Schwierigkeiten bei ihr zu finden.

Zunächst betrachtet sie das Wort simplaria als perfektes Synonym für simplex, was unklar lässt, weshalb Pomponius genau dieses merkwürdige, sonst nie (insofern uns überliefert) verwendete Wort ausgewählt hat, und gerade um eine so triviale Anmerkung zu machen, wie die herrschende Meinung behauptet: wird die Wandelung ausdrücklich ausgeschlossen, greift sie keinen Platz. Dieses Wort müsste dagegen eine sehr spezifische Bedeutung haben, und sogar eine Bedeutung, die wahrscheinlich genau für die Stelle durch eine bewusste grammatikalische Verwandlung konzipiert worden ist — als Hapaxlegomenon müsste es doch auch ein Neologismus sein.

Des Weiteren bleibt der Hinweis auf eine Gewohnheit (in usu est) unerklärt. Wenn es in der Stelle um den ausdrücklichen Ausschluss der Wandelung geht, ist die Erwähnung des usus überflüssig, oder sogar widersprüchlich: es wäre nicht die Gewohnheit, sondern eben die vertragliche Vereinbarung des Ausschlusses, die die Wandelung ausschließen würde. Die einzige mögliche Lösung dieses Widerspruches der herrschenden Meinung wäre die Bezugnahme auf den usus so zu verstehen, dass Pomponius eigentlich gesagt hätte: es ist gängige Praxis den Kauf als simplaria venditio zu gestalten, sodass die Wandelung ausgeschlossen wird. Das sagt aber der Wortlaut des Textes eben nicht: was üblich ist, ist die Tatsache, dass, wenn es sich um eine simplaria venditio handelt, dann gibt es keine Wandelung. Wenn die simplaria venditio als „Kauf mit ausdrücklichem Ausschluss der Wandelung“ angenommen wird, enthält die Stelle nach ihrem Wortlaut gelesen einen Widerspruch (was vertraglich vereinbart wurde, kann nicht ,,aus Gewohnheit“، gelten).

Als Alternativ lässt es sich denken, dass Pomponius etwas ganz Spezifisches in der Stelle gesagt hätte, das nicht so sehr mit den vorigen Stellen in D. 21, 1, 48 zu

15 Quod autem diximus duplam promitti oportere, sic erit accipiendum, ut non ex omni re id accipiamus, sed de his rebus, quae pretiosiores essent, si margarita forte aut ornamenta pretiosa vel vestis serica vel quid aliud non contemptibile veneat. Per edictum autem curulium etiam de servo cavere venditor iubetur. 
tun hätte (was übrigens nicht zu verwundern wäre, da die Stelle offenbar außer Kontext von den Kompilatoren gestellt wurde). Die Rede könnte nämlich von einem Kauf sein, der unter Abschluss der stipulatio simplae statt der duplae für den Fall der Eviktion vereinbart würde. Nicht aber der Kauf, bei dem und wegen dessen Gegenstands keine stipulatio duplae hätte gefordert werden können, sondern der Kauf, bei dem die Parteien freiwillig eine Minderung der Haftung für Rechtsmängelhaftung vereinbaren würden, wie es in D. 21, 2, 56 pr. erwähnt wird. Die Logik wäre dann, dass die freiwillige Minderung der Rechtsmängelhaftung üblicherweise von einer Minderung der Sachmängelhaftung begleitet würde, weshalb es üblicherweise keine Wandelung Platz griffe. ${ }^{16}$ Das kann entweder heißen, dass die tatsächliche gängige Praxis auf dem Markt so war, dass die Parteien meistens auch eine Minderung der Sachmängelhaftung freiwillig vereinbarten, oder vielmehr, dass das Schweigen über Sachmängelhaftung bei einem Kauf mit Minderung der Rechtsmängelhaftung (stipulatio simplae statt duplae) üblicherweise auch als eine Minderung der Sachmängelhaftung (Ausschluss der Wandelung) vom Richter interpretiert wurde, im Sinne einer juristischen Vermutung oder einer ergänzenden Vertragsauslegung. Beide Möglichkeiten schließen sich gegenseitig nicht aus: Die erste (eine tatsächliche gängige Praxis) könnte sich in die zweite (juristische Vermutung/ ergänzende Vertragsauslegung) entwickelt haben.

Natürlich bleibt diese Hypothese insoweit nur eine Konjektur; die Stelle allein lässt sie aus sich nicht entnehmen. Als Versuch, diese Vermutung unter Beweis zu stellen, oder zumindest ihre Wahrscheinlichkeit zu zeigen, werden jetzt einige Quellen analysiert, die irgendwie mit der simplaria venditio zu tun haben könnten. In keiner von ihnen taucht das lateinische Wort ,simplaria“ auf (es ist, wie gesagt, ein Hapaxlegomenon), aber es ist doch in ihnen von ,simpliciter emere/vendere“ die Rede, oder sie sind normalerweise von der Literatur mit der simplaria venditio verbunden worden.

Die zu analysierenden Stellen sind nämlich: der Papyrus P. Cair. Preis. 1; die Konstitution C. 4, 58, 1 des Kaisers Antoninus Severus (Caracalla); die Paragrafen $\S \S 39 \mathrm{~b}$ und $113 \mathrm{~b}$ des syrisch-römischen Rechtsbuches; ein Scholion des Stephanus zu Bas. 18, 6, 2 und dazu noch TH 61 und FIRA III 132.

16 Nichts wird in D. 21, 1, 48, 8 über die Preisminderung durch die actio quanti minoris gesagt, was streng genommen die Hypothese der Minderung der Sachmängelhaftung als Begleiterscheinung auf die Wandelung begrenzt. Die Literatur jedoch, besonders die Vertreter der herrschenden Meinung, geht davon aus, es stehe außer Zweifel, die Preisminderung sei in D. 21, 1, 48, 8 implizit gemeint. Vgl. dazu L. Wenger, (1953, p. 778). Dagegen: P. Meyer (1921, p. 258). 
II. Analyse der Quellen

1. P. Cair. Preis. (2) 1: Zusammenhang zwischen der simplaria venditio und der simpla pecunia.

Die erste $\mathrm{zu}$ analysierende Quelle ist ein in Bacchias gefundenes, wahrscheinlich aber aus Oxyrhynchus stammendes Papyrus, das irgendwann zwischen 147 und 150 n. Chr. — also wenige Jahre nach der Zeit Pomponius — verfasst worden sein soll. Es geht um eine Sklavin namens Sarapiona, die ihren Herrn bestohlen hat und danach geflohen ist. Der Herr will jetzt die Rückgabe des Preises vom Verkäufer (Wandelung), ${ }^{17}$ der die Sklavin verkauft hatte. Der Anwalt des Verkäufers, Lycarion, zitiert jedoch einen Brief vom Behörden Honoratus, um die Meinung drei iuridici zu widerlegen, denen zufolge der Preis zurückgegeben werden müsse. Die Argumentation beruht auf die Tatsache, dass der Käufer $\dot{\alpha} \pi \lambda \tilde{\omega} \chi \chi \eta \dot{\mu} \mu \alpha \tau$ (simpla pecunia) gekauft habe, weshalb der Verkäufer nicht bei einem solchen Prozess (um die Rückgabe des Preises wegen Sachmängel, d.h. Wandelung) haftbar sei. Relevant sind hier die Zeilen 14 bis 20, die nach der 2. Auflage der P. Cair. Preis. von 2014 folgen:

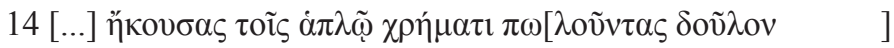

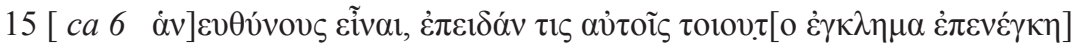

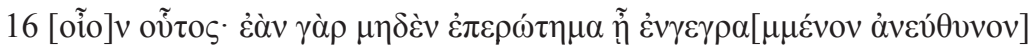

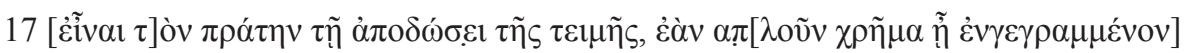

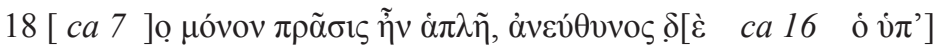

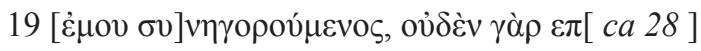

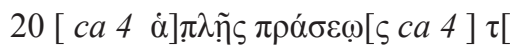

Was folgenderweise übersetzt werden könnte:

14 [...] Du hast gehört, dass diejenigen, die mit einfachem Geld [einen Sklaven kaufen]

15 [ ca 6 un] haftbar sind, wenn jemand gegen sie eine [Klage einreicht]

16 [wie] diese; wenn nämlich keine Stipulation hinzu[gefügt worden ist, ist unhaftbar]

17 [d]er Verkäufer, den Preis zurückzugeben; wenn ein[faches Geld hinzugefügt worden ist]

17 Auf der Z. 9 ist zwar von der Rückgabe des Preises und der gestohlenen Sachen (deren Wert) die Rede, also von Schadensersatz - worin der Gegenstand der Wandelung nicht besteht. Da aber der Teil über die Rückgabe der gestohlenen Sachen nur vermutet wird (das Papyrus ist da unlesbar), und auf der Z. 17 (die wirklich zur zentralen Argumentation gehört und deswegen hier wichtig ist) nur die Rückgabe des Preises erwähnt wird, wird hier davon ausgegangen, dass es tatsächlich um die Wandelung geht. Zur Annahme, die ädilizische Klage sei (mindestens im Wesentlichen) in den Provinzen bekannt gewesen, sodass hier von der actio redhibitoria die Rede sein könne, vgl. auch É. Jakab (2018, p. 489 et seq.). 
18 [ ca 7 nicht] nur war der Kauf einfach, sondern au[ch ca 16 mein]

19 [Ku]nde unhaftbar, denn nichts [ $\mathrm{ca} 28$ ]

20 [ ca 4 e]infachen Kauf[es

Zunächst muss der Ausdruck „, $\alpha \lambda \tilde{\omega} \chi \rho \eta ́ \mu \alpha \tau \imath$ “ aufZ. 14 kommentiert werden. Wörtlichübersetztheißtes, ,simplapecunia“aufLatein, also: „,mit einfachem Geld“. Obwohl nicht alle damit einverstanden sind, ${ }^{18}$ ist diese Ausdrucksform höchstwahrscheinlich als „unter Abschluss der stipulatio simplae für Eviktionsfall““ zu lesen. ${ }^{19}$ Wie kann es aber

18 Vgl. F. Pringsheim (1950, p. 483).

19 Vgl. J. F. Gilliam (1971, p. 70). Dagegen É. Jakab (1997, p. 207). Sie behauptet, F. Pringsheim (1950, p. 483) habe diese These schon völlig überzeugend widerlegt. Ein Blick auf die betroffenen Papyri P. Col. 8219 [= SB 12 10894] (Alexandria 140 n. Chr.), P. Freib. II 8 (? nach 144 n. Chr.), SB VI 9145 [= P. Vindob. g. Inv. 25817] (Arsinoites 184-192 n. Chr.), P. Oxy. XLI 2951 (Oxyrhynchus 267 n. Chr.) und P. Ryl. IV 709 (? 294-296 n. Chr.) — zeigt aber, dass die These doch zumindest möglich ist. Sie lassen sich nämlich so interpretieren, dass mit den Worten , $\dot{\alpha} \pi \lambda \tilde{\varphi} \chi \rho \eta \dot{\mu} \mu \alpha \iota^{\prime \prime}$ die Rede vom Abschluss einer stipulatio simplae für den Eviktionsfall zwischen dem Verkäufer und demjenigen, der ihm zuvor verkauft hatte, wäre. Vlg. J. Partsch, P. Feib. II, 28-29. Der Ausdruck kommt immer inmitten der Deskription

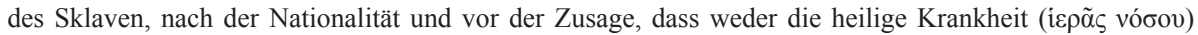
noch Aussatz $(\dot{\varepsilon} \pi \alpha \varphi \tilde{\eta} \varsigma)$ vorliegt. Interessanterweise wird diese letzte Zusage nicht mit der konzessiven

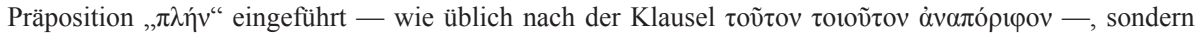

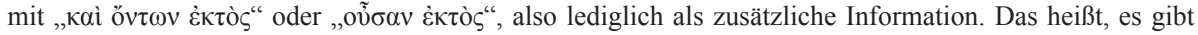
keine notwendige Opposition zwischen $\dot{\alpha} \pi \lambda \tilde{\omega} \chi \rho \eta \dot{\eta} \mu \alpha \tau$ und dieser Zusage, als ob sie eine Einschränkung des Ausschlusses der Haftung für Sachmängel wäre, nach den typischen hellenistischen Ausnahmen. Es scheint vielmehr so zu verstehen zu sein, dass eine Reihe an Informationen über den Sklaven vermittelt wird: nach der Mitteilung der Nationalität und manchmal des Alters wird gesagt, dass der Verkäufer den Sklaven mit Garantie (simpla pecunia) gekauft hatte, was jetzt als Zusicherung seines Eigentumsrechts dienen kann, und darüber hinaus wird auch gesagt — was der Käufer immer hören will —, dass weder die heilige Krankheit noch Aussatz vorliegt. Die Widerlegung dieser Möglichkeit für P. Freib. II 8 durch F. Pringsheim (1950, p. 483) klingt nicht hundertprozentig überzeugend: Ihm zufolge habe eine Zusicherung des Eigentumsrechts des Verkäufers im Fall keinen Sinn, weil er den Sklaven geerbt, und nicht gekauft habe. Nichts verhindert aber, dass mit $\dot{\alpha} \lambda \hat{\tilde{\omega}} \chi \rho \eta \dot{\mu} \alpha \tau$ die Rede doch vom letzten Kauf des Sklaven (also, mit dem der Erblasser den Sklaven gekauft hatte) ist, denn das gilt indirekt als Eigentumsbeweis für den Verkäufer. Bezüglich

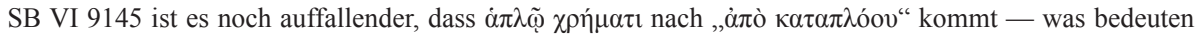
soll, der Sklave wurde sofort nach seiner Ankunft in Ägypten erworben (vgl. LEWALD, 1953, p. 435). Es wäre also nicht zu verwundern, dass eine andere Information (Kauf simpla pecunia) über den Erwerb des Sklaven zugleich gegeben wäre. In P. Oxy. XLI 2951 ist auf Latein die Beurkundung der stipulatio simplae für den beurkundeten Kauf (WOLFF, 2002, p. 139), aber auf Griechisch wiederum wahrscheinlich den Bezug auf den vorigen Kauf (nach der Mitteilung der Nationalität). P. Ryl. IV 709 ist zu fragmentiert um

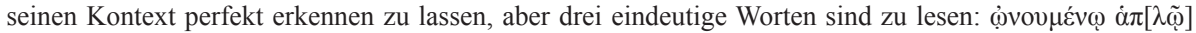
$\chi \rho \eta ́ \mu \alpha \tau$, der Sklave ist „gekauft mit einfachem Geld“. Obwohl der Partizip praesens auf Griechisch zu lesen ist, der Hinweis auf die Garantie für Eviktion des beurkundeten Kaufes auf Z. 10 ist ein Grund dafür, dass auf Z. 6 die Rede doch vom vorigen Kauf ist (keine Klausel pflegte zweimal geschrieben zu werden — PRINGSHEIM, 1950, p. 467). Dafür spricht auch, dass der Ausdruck „ $\delta \imath \pi \lambda \tilde{\omega} \chi \rho \eta \dot{\mu} \mu \tau \imath^{\prime \prime}$ in einem anderen Papyrus (P. Eitrem. 5 = SB 3 6016, Alexandria 154 n. Chr.) auf eine sehr ähnliche Weise verwendet wird: In der Deskription des Sklaven, nachdem es gesagt wurde, wie der Sklave gekauft worden war, und vor den Zusagen, der Sklave sei vertraulich, nicht zur Flucht geneigt, und nicht von der heiligen Krankheit oder Aussatz affektiert. Der Gegensatz zwischen $\delta \imath \pi \lambda \tilde{\omega}$ und $\dot{\alpha} \pi \lambda \tilde{\omega}$ ist ein starkes Argument für die Übersetzung als simpla pecunia im Sinne der stipulatio simplae, die der duplae gegenübersteht (dupla pecunia kann kaum etwas anders als ,unter Abschluss der stipulatio duplae für Rechtsmängelhaftung“ bedeuten). Die zusätzlichen positiven Zusagen, dass der Sklave vertraulich und kein fugitivus ist, sprechen auch dafür, 


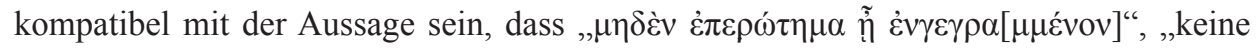
Stipulation ${ }^{20}$ zugefügt wurde“"? Eben dadurch, dass nur auf Z. 16 von der Stipulation für Sachmängelhaftung die Rede ist. Das heißt: hat der Käufer mit der stipulatio simplae für Rechtsmängelhaftung, ohne aber eine Stipulation für Sachmängelhaftung, den Sklaven

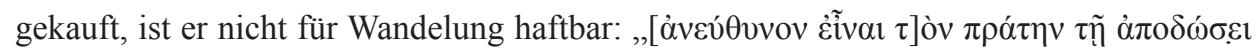

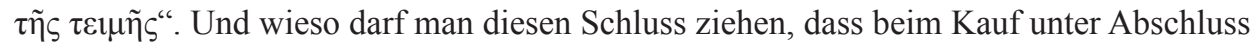
der stipulatio simplae die Wandelung, soweit es keine Stipulation für Sachmängel gab, ausgeschlossen war? Das Ädilenedikt galt wahrscheinlich nicht außerhalb Rom, aber die Prinzipien der Haftung für Rechtsmängel ohne ausdrückliche Stipulation musste doch auch sogar im romanisierten Ägypten verbreitet sein, auch wenn ihren Ausschluss häufig vereinbart wurde (PRINGSHEIM, 1950, p. 486) ${ }^{21}$ (sonst wäre die ganze Argumentation des Papyrus in Betrachtung eigentlich überflüssig gewesen). Diese Haftung ist im Fall aber eben und nur deswegen ausgeschlossen, weil der Sklave „simpla“ statt wie üblich „,dupla pecunia“ für Rechtsmängel gekauft wurde. Der Kauf mit „simpla pecunia“ ist eine

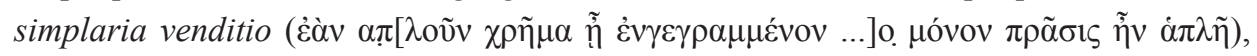
und, bei solchen Käufen — solange es keine Stipulation für Sachmängel gab — ist der

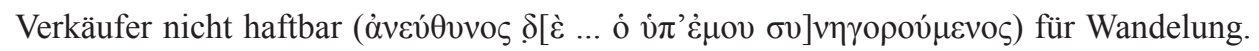
Diese Verbindung von Rechts- und Sachmängelhaftung muss genau diejenige sein, die Pomponius in D. 21, 1, 48, 8 feststellt, wenn man in der „simplaria venditio“ den Kauf unter Abschluss der stipulatio simplae sieht; und diese Verbindung wäre nach Pomponius auf die Gewohnheit zurückzuführen (in usu est).

Auf jeden Fall scheint diese Interpretation der Ausdrücke , $\dot{\alpha} \pi \lambda \tilde{\omega} \chi \rho \eta \dot{\mu} \mu \alpha \tau \iota^{\prime \prime}$ und , $\dot{\alpha} \pi \lambda \tilde{\eta} \pi \rho \tilde{\alpha} \sigma 1 \varsigma^{\prime \prime}$ in P. Cair. Preis. 1 und des Ausdrucks „simplaria venditio“ bei Pomponius viel befriedigender als die Auslegung, die die simplaria venditio für den Kauf

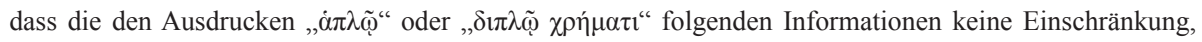
sondern einfach zusätzlich sind. Letztlich kann auch SB 1813.173 (Hermopolis, 629 n. C.) erwähnt werden, als ein später Beweis der Möglichkeit einer $\dot{\alpha} \pi \lambda \tilde{\eta}$ ஸ்vท́ (Z. 21-22) mit Haftung für Sachmängelhaftung (Z. 28-34), d.h. ein Beweis dafür, dass sogar nach Justinian der einfache Kauf nicht mit vertragsmäßigem

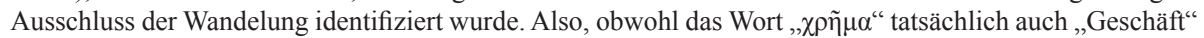
bedeuten kann, und insofern in allen erwähnten Papyri die Rede (rein grammatikalisch gesehen) von einem „einfachen Geschäft“ im Sinne von „einfachem Kauf“ sein könnte, ist es auch (und von dem Kontext her eher) doch vorstellbar, das in ihnen $\dot{\alpha} \pi \lambda \tilde{\omega}$ $\chi \rho \hat{\mu} \mu \alpha \tau$ f für simpla pecunia (=mit Abschluss der stipulatio simplae für Rechtsmängelhaftung) steht. In P. Cair. Preis. 1 hätte der Ausdruck daher dieselbe Bedeutung. Zur ausführlichen Analyse der Sachverhalte dieser Urkunden vgl. É. Jakab (2018). Die Verfasserin geht ihrer schon früher (1997) aufgestellten Auffassung entsprechend davon aus, $\dot{\alpha} \pi \lambda \tilde{\omega} \chi \rho \eta \dot{\mu} \mu \alpha \tau$ beziehe sich direkt auf den Ausschluss der Wandelung, doch die eben dargestellten Überlegungen bleiben trotz der Meinung Jakabs weiterhin zumindest als alternative Auslegung möglich. Apropos: die Tatsache, dass alle erwähnten Papyri römische Bürger als mindestens eine der Parteien haben, reicht, um anzunehmen, dass römisches Recht in ihnen tatsächlich widerspiegelt wird (URBANIK, 2010, p. 230).

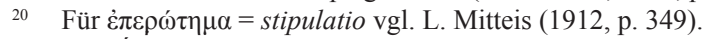

21 Vgl. É. Jakab (2018). 
mit ausdrücklichem Ausschluss der Wandelung hält. Wenn so was der Fall wäre, wäre es für Lycarion sinnlos gewesen, sowohl die Unterlassung der Stipulation für Sachmängelhaftung zu erwähnen (denn es gäbe diesbezüglich schon eigentlich ein negatives Abkommen, es

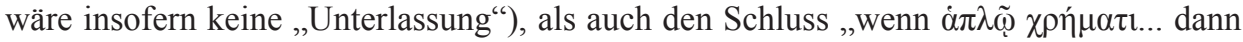
$\dot{\alpha} \pi \lambda \tilde{\eta} \pi \rho \tilde{\alpha} \sigma 1 \varsigma^{\prime \prime}(Z .17-18)$ zu ziehen, es wäre einfach redundant gewesen.

Dass die anderen oben erwähnten Meinungen zur Auslegung von D. 21, 1, 48, 8 nichts mit diesem Papyrus haben können, liegt auf der Hand. Beide hängen nämlich vom Gegenstand des Kaufs ab: Die simplaria venditio wäre entweder der Kauf geringwertiger Sachen oder der Kauf, bei dem (wegen seines Gegenstandes) keine stipulatio duplae erforderlich war. Im Papyrus geht es aber gar nicht um die Kaufsache (eine Sklavin), sondern vielmehr um die simpla pecunia, um die geschäftlichen Bedingungen des Kaufs.

\section{Die Kaiserkonstitution C. 4, 58, 1 und deren eigentlicher Sinn}

Von zentraler Bedeutung zur Lösung des Problems ist die Kaiserkonstitution C. 4, 58, 1 des Imperators Antoninus Severus (Caracalla), die aus dem Jahr 214 n. Chr. stammt. Folgt der Text der Konstitution:

Si non simpliciter, sed concilio fraudis servum tibi nescienti fugitivum vel alio modo vitiosum quis vendidit isque fugitivus abest, non solum in pretio servi venditorem conveniri, sed etiam damnum quod per eum tibi accidit competens iudex, ut iam pridem placuit, praestari iubebit. ${ }^{22}$

Es geht um einen abwesenden flüchtigen Sklaven, der fugitivum oder anders mangelhaft verkauft wurde, und zwar non simpliciter, sed concilio fraudis. Der Kaiser sagt, der Verkäufer nicht nur um den Preis des Sklaven, sondern auch um den Wert des dem Käufer zugefügten Schadens verurteilt werden soll, wie es schon früher verstanden wurde (ut iam pridem placuit).

Die Stelle wird häufig, wie z.B. von É. Jakab (1997, p. 218 et seq.), folgenderweise verstanden: es werde nicht mit ausdrücklichem Ausschluss der Wandelung verkauft, d.h. nicht als simplaria venditio (si non simpliciter), sondern mit positiven Zusagen, dass der Sklave fehlerfrei sei, sodass es um einen Kauf bonis condicionibus handle (was mit sed concilio fraudis gemeint sei, da der Vorsatz des Verkäufers zur Täuschung des Käufers die positiven Zusagen voraussetze, die einen Kauf bonis condicionibus gestalte). Da der Kauf bonis condicionibus abgeschlossen worden sei,

\footnotetext{
22 „Wenn Dir jemand einen Sklaven, der, ohne dass Du es wusste, Ausreißer oder auf andere Weise mangelhaft ist, nicht in gutem Glauben (simpliciter), sondern mit Vorsatz (concilio fraudis) verkauft hat, und der Sklave geflohen und jetzt abwesend ist, soll der Verkäufer nicht nur auf den Preis des Sklaven, sondern auch auf Schadensersatz verurteilt werden, wie es schon früher entschieden wurde (ut iam pridem placuit)“.
} 
bekomme der getäuschte Käufer den Preis des Sklaven plus den Wert des Schadens, den der Sklave dem Käufer eventuell zugefügt habe (beim Diebstahl vor der Flucht usw). ${ }^{23}$

Diese Auslegung ist aber doch nicht unproblematisch. Die Haftung für Zusicherungen war gemäß dem Ädilenedikt die redhibitio (Rückgabe des Preises) oder die Preisminderung. Der Schadensersatz war prinzipiell nicht versprochen. ${ }^{24}$ Der Verkäufer war dann, und nur dann um Schadensersatz verurteilt zu werden, wenn der Käufer entweder mit der actio ex stipulatu oder mit der actio empti klagte. In der Konstitution C. 4, 58, 1 wird ja den Schadensersatz versprochen, und die Bedingung dazu ist, dass der Sklave non simpliciter, sed concilio fraudis verkauft wurde. Concilium fraudis bzw. dolus malus war völlig irrelevant für die actio ex stipulatu, ein iudicium stricti iuris. Es war hingegen für die actio empti, ein iudicium bonae fidei, extrem relevant: es könnte für den Richter eben der Grund der Verurteilung sein, und zwar nicht nur zur Rückgabe des Preises, sondern auch zum Schadensersatz. In der Konstitution muss also von den Folgen der actio empti die Rede sein. Das ist zwar nichts Neues, wie die Anmerkung ,ut iam pridem placuit“ übrigens erwarten lässt. Der Kaiser hat hier keine neue Regel erfunden. Er hat einfach festgestellt, dass eine bestimmte Situation mit der vom Prätor schon früher erfundenen Mitteln zu lösen war, was in einer Kaiserkonstitution übrigens nicht zu verwundern ist (KASER; KNÜTEL; LOHSSE, 2017, p. 29). Obwohl die Konstitution unter dem Titulus „De aediliciis actionibus“ im Codex steht, heißt das nicht, dass der Kaiser Caracalla tatsächlich über die ädilizischen Klagen gesprochen hatte, sondern lediglich dass die Kompilatoren ihn so (und nicht notwendig richtig) verstanden haben. Davon wird aber bald die Rede sein.

Auf jeden Fall ist also simpliciter hier im Gegensatz zu „,consilio fraudis“ verwendet worden: ohne Vorsatz, im guten Glauben. Insofern hat es überhaupt nichts mit der simplaria venditio von D. 21, 1, 48, 8 zu tun, außer der zufälligen Verwandtschaft der Worten ,simpliciter“ und ,simplaria“. Diese zufällige Verwandtschaft wird aber relevant sein, um die nächste Quellen zu analysieren.

23 Eine andere Auslegung der Stelle wird von G. Impallomeni (1955, p. 35) vorgeschlagen, um simpliciter als simplaria venditione zu lesen: der dolus malus wäre inkompatibel mit der simplaria venditio, denn für dolus haftet der Verkäufer auf jeden Fall (,siccome il dolo esclude l'efficacia di qualsiasi patto di non assunzione di garanzia per i vizi occulti, la compravendita conclusa da un venditore in mala fede non può essere considerata simplaria“). Aber für dolus haftet der Verkäufer schon mit der actio empti, und nicht mit der ädilizischen Klage, die eventuell vertragsmäßig ausgeschlossen worden sein konnte.

24 Er konnte nur durch retentio erreicht werden (D. 21, 1, 29, 3 Ulp. 1 ed. aedil. curul.), aber nie aktiv verlangt. Der Verkäufer wurde nie durch actio redhibitoria dazu ,verurteilt““. 
3. SRR $\S \S 39 \mathrm{~b}$ und $113 \mathrm{~b}$ und Scholion des Stephanus: Ursprung einer verschiedenen Auslegung

Die nächsten zu analysierenden Stellen sind die Paragraphen 39 und 113 des syrisch-römischen Rechtsbuches bzw. der zweite Teil jeweils, und ein Scholion des Antecessors Stephanus zu Basiliken 18, 6, 2. In beidem ist ein Phänomen zu entdecken, das mit dem Zeitpunkt der Entstehung dieser Stelle zu tun haben kann. Jetzt ist zwar von einer $\dot{\alpha} \pi \lambda \tilde{\eta} \dot{\omega} v \eta \dot{~(e i n f a c h e r ~ K a u f) ~ u n d ~ v o n ~} \dot{\alpha} \pi \lambda \tilde{\omega} \zeta$ (einfach) Kaufen die Rede, aber durch eine aufmerksame Analyse wird es klar, dass sie wenig mit der simplaria venditio von D. 21, 1, 48, 8 zu tun haben können. Der „einfache Kauf“ wird hier auf eine Weise verstanden, die nicht mit dem Wortlaut vom Pomponiusfragment vereinbar ist. In der sprachlichen Formulierung der Quellen lässt sich aber beobachten, was eine Spur des ursprünglichen Sinnes sein könnte.

Zuerst werden die Paragraphen des SRR analysiert. Das Buch ist wahrscheinlich ein am Ende des 5. Jh. (474 n. Chr.) verfasstes Unterrichtmaterial, das hauptsächlich zur Erklärung von Kaiserkonstitutionen gedient hat. Es wurde vermutlich in Beirut und Antiochia benutzt und widerspiegelt zwar römisches (und kein syrisches, trotz des Titels), auch wenn nicht klassisches Recht (SELB; KAUFHOLD, 2002, p. 3-5). ${ }^{25}$ Die hellenistische Denkweise der Provinzen ist aber häufig in ihm zu erkennen.

Die folgende ist die durch M. Memmer (1990, p. 3-5) ${ }^{26}$ vorgeschlagene Übersetzung für die Stellen, die hier relevant sind:
$\S 39 \mathrm{~b}:$
Wenn er aber einen jungen Mann als Sklaven ,einfach“ kauft, ob er (der Sklave) nun gut oder schlecht sei, mit der Abrede, dass keiner zurückgibt seinem Partner, was auf griechisch heißt $\dot{\alpha} \pi \lambda \tilde{\eta} \dot{\omega} v \dot{\eta}$, und wenn dann der, der den Sklaven gekauft hat, ihn dem, der ihn verkauft hat, zurückgeben will, so erlaubt es ihm das Gesetz nicht, dass er ihn zurückgebe. Er kann ihn nicht zurückgeben, außer wenn ein Dämon in dem Sklaven gefunden wird. Wenn sich aber ein Dämon in dem Sklaven zeigt und wenn er ihn dem, der ihn verkauft hat, zurückgeben will, ist es ihm erlaubt, dass er ihn zurückgebe.

\footnotetext{
25 Vgl. É. Jakab (1997, p. 212).

26 Da der Verfasser sich auf diese zwei Paragraphen fokussiert hat, eben um die Sachmängelhaftung zu besprechen, und da seine Übersetzung klarer, obwohl nicht im Wesentlichen unterschiedlich von der Übersetzung Selbs (SRR II, 60-61 [§37] und 144-145 [§101]), für den Zweck dieser Analyse ist, ist sie hier ausgewählt worden.
} 
$\S 113 b$ :

(I) Wenn aber ein Mann einen Sklaven oder eine Sklavin

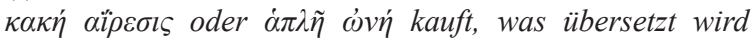
als „schlechter Vertrag“ oder ,einfacher Kauf" ohne Rückgabe, und dann der Mann, der ihn gekauft hat, eben den Sklaven oder die Sklavin zurückgeben will, so darf er (es) nicht, weil er ihn mit einem „schlechten Vertrag“ gekauft hat. Wenn er aber in eben dem Sklaven oder der Sklavin einen Dämon findet, so ist es ihm erlaubt, dass er ihn (R I 1 oder sie) dem, der ihn verkauft hat, zurückgibt und sein Geld nimmt.

(II) Wenn es sich aber ereignet, dass der junge Mann oder die Sklavin flüchtig zum Hause ihrer früheren Herren und ein Dieb ist, dann darf der Käufer sie, den jungen Mann oder die Sklavin, zurückgeben. Und nicht nur das; sondern es kann der Mann von dem, der den Sklaven verkauft hat, auch die $\tau$ un verlangen, d.h. das was er gestohlen hat. Aber nur

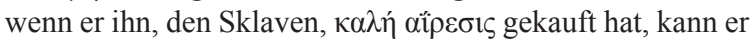
dies fordern. Wenn er ihn, den Sklaven, aber in ,schlechtem Vertrag" gekauft hat, so kann er das nicht. Und er gibt ihn weder zurück noch verlangt er etwas, was er gestohlen hat.

In beidem geht es um die Unmöglichkeit der Wandelung wegen einer konkreten Gestaltung des Kaufs.

Im $\S 39$ b ist von einer $\dot{\alpha} \pi \lambda \tilde{\eta} \omega \dot{\omega} \eta \dot{~ d i e ~ R e d e, ~ d e r ~ „ e i n f a c h e ~ K a u f, ~ o b ~ d e r ~}$ Sklave nun gut oder schlecht sei, mit der Abrede, dass keiner zurückgibt seinem Partner“. Bei einem solchen Kauf erlaube das Gesetz nicht, dass der Sklave zurückgegeben wird, es sein denn, dass „ein Dämon in ihm gefunden wird“ (die typische hellenistische Ausnahmeklausel). Im $\S 113 b$ wird $\dot{\alpha} \pi \lambda \tilde{\eta} \dot{\omega} v \eta ́$ als „einfacher Kauf ohne Rückgabe“ übersetzt. Bei so einem Kauf, der auch ,,schlechter Vertrag“" genannt wird, darf der Käufer den Sklaven nicht zurückgeben, und auch wenn der Sklave ihn gestohlen hat und geflohen ist, darf er weder den Preis noch den Wert der gestohlenen Sachen verlangen.

Auf den ersten Blick könnte man vermuten, dass die $\dot{\alpha} \pi \lambda \tilde{\eta} \omega \dot{v} \eta \dot{~ h i e r ~}$ identisch mit der simplaria venditio von D. 21, 1, 48, 8 (Pomp.) wäre. ${ }^{27}$ Dass es aber keine direkte Verbindung zwischen ihnen gestellt werden darf, zeigt schon der hier gegebene Grund, warum der Käufer den Sklaven nicht zurückgeben darf: Das Gesetz erlaube es ihm nicht — wogegen Pomponius schrieb, es sei ,in usu“, dass die Wandelung bei simplariae venditiones keinen Platz greife. Gesetz und Gewohnheit sind zwei verschiedene Gründe für die Rechtsfolge, die verschiedene Begriffe für den „einfachen Kauf“ erkennen lassen.

27 Vgl. z.B. É. Jakab (1997); B. Kupisch (2002); P. Meyer (1921). 
Also, die $\dot{\alpha} \pi \lambda \tilde{\eta}$ ஸ̉vท́ des SRR ist als der Kauf angesehen, bei dem es weder Rückgabe des Preises noch Schadensersatz möglich ist — was als gesetzliche Bestimmung gekennzeichnet wird. Was für ein Gesetz könnte hier gemeint sein? Vielleicht eben die oben erörterte Kaiserkonstitution C. 4, 58, 1: Es könnte sich um eine falsche Auslegung der Konstitution handeln, indem sie a contrario sensu interpretiert wurde, aber mit dem durch É. Jakab vorgeschlagenen Hintergrund: Das „si non simpliciter, sed concilio fraudis“ im Text der Konstitution könnte hier so verstanden worden sein, dass ,simpliciter" für „mit vertraglichem Ausschluss der Wandelung“ stünde, und „,concilio fraudis“ einen Kauf „bonis condicionibus" voraussetzte. In den $\S \S 39 \mathrm{~b}$ und $113 \mathrm{~b}$ könnte also eine Auslegung $a$ contrario sensu der C. 4, 58, 1 vorliegen, die auf einer falschen Interpretation des Wortes „simpliciter“ beruhte. Bei den Käufen mit Abrede der Rückgabe ( $\dot{\alpha} \pi \lambda \tilde{\eta} \omega \dot{\omega} v$ ), erlaube das Gesetz keine Rückgabe und keinen Schadensersatz.

Aber woher wäre diese falsche Interpretation gekommen? Das kann man nicht genau wissen, aber es lässt sich doch vermuten, dass in der langen Periode zwischen der Auffassung des 23. Buches ad Sabinum des Pomponius und der Entstehung des spätantiken SRR eine allmähliche Verwandlung des Verständnisses der simplaria venditio stattgefunden hätte. Die Tatsache, dass sie üblicherweise mit dem Ausschluss der Wandelung verbunden war (D. 21, 1, 48, 8), was nach der vorgeschlagenen Auslegung der Pomponiusstelle sogar als Basis für eine Vermutung im juristischen Sinne (presumptio) gewirkt hat, könnte nach und nach zu einer Identifizierung der zwei ursprünglich verschiedenen Dinge geführt haben: der Name „simplaria venditio“ (wegen Abschlusses der stipulatio simplae statt duplae für Rechtsmängel) und der Ausschluss der Wandelung. Durch Metonymie pars pro toto könnte ,simplaria venditio“ zuerst für das ganze Komplex „Kauf unter Abschluss der stipulatio simplae für Rechtsmängel und aus allgemeiner Gewohnheit prinzipiell vermuteter Ausschluss der Wandelung“ gestanden haben, und dann nur für die Folge: Kauf mit Ausschluss der Wandelung. Eine Spur davon könnte die im SRR zu lesende merkwürdige redundante Ausdrucksform ,einfacher Kauf... mit Abrede“ bzw. „einfacher Kauf ohne Rückgabe“ sein.

Dieser Verwandlungsvorgang der Bedeutung von „simplaria venditio“ hätte nicht die Kaiserkonstitution C. 4, 58, 1 beeinflusst, aber doch ihre Auslegung ab dem 5. Jh. Ähnliches lässt sich auch im Scholion zu den Basiliken beobachten, das unten analysiert wird.

Das im 6. Jh. entstandene Scholion vom Antecessor Stephanus ist ein Versuch, einen Überblick der verschiedenen möglichen Gestaltungen der Haftung für Sachmängelhaftung nach dem Ädilenedikt und der entsprechenden Fristen (problematisch wegen D. 21, 1, 28 Gai.) zu gewinnen. É. Jakab (2009) hat die Zuverlässigkeit der Stelle streng bestreitet, als sie die Argumente B. Kupischs gegen ihre Theorie zum prozessualen Mechanismus des Edikts widerlegt hat. Es handle sich wahrscheinlich um Notizen, die 
ein fleißiger Student aufgeschrieben habe, als sein Professor Stephanus eine typische byzantinische didaktische Simplifizierung eines komplexen klassischen Phänomens erklärt habe. So lautet das Scholion:

Si quis servum vendiderit, qui vitium latens habet, emtori competit actio aedilicia redhibitoria, vel quanti minoris servum emisset emtor, si vitium cognitum habuisset. Et redhibitoria quidem dumtaxat intra sex menses agitur, quanto minoris autem intra anum utilem, si nimirum venditor emtori caverit de his, quae edicto aedilium continentur, etenim si ei non caverit, redhibitoria dumtaxat intra duos menses, quanti minoris intra sex menses agitur, ut Gaius dig. 28 dicti lib. et tit. librorum singularium Antipapiniani [D. 21, 1, 28] dicit. Sed hoc sic accipe, si non simpliciter et ita, ne restitutio fieret venditor servum vendiderit: nam adversus eum, qui simpliciter et ita, ne restitutio fiat, vendit, aediliciae actioni non est locus, ut Papinianus (sic) dicit dig. 48 eiusdem lib. et. tit. [D. $21,1,48,8]^{28}$

Und der letzte Teil (Sed hoc sic accipe...) im griechischen Original:

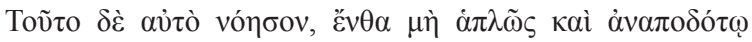

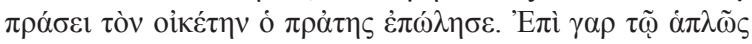

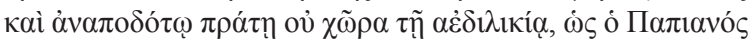

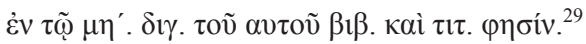

Stephanus erklärt die Sachmängelhaftung beim Ädilenedikt so, dass es drei verschiedene mögliche Gestaltungen der Haftung gebe: $i$. Der Käufer könne sich durch eine Stipulation versprechen lassen, dass die im Edikt erwähnten Mängel nicht zutreffen (si nimirum venditor emtori caverit de his, quae edicto aedilium continentur); ii. Er könne ebenso keine Stipulation abschließen (si ei non caverit), mit einer Verkürzung der Fristen für die redhibitoria als Folge; iii. Er dürfe aber auch den Sklaven simpliciter et ita, ne restitutio fieret ( $\dot{\alpha} \pi \lambda \tilde{\omega} \varsigma \kappa \alpha i ̀ ~ \alpha ่ v \alpha \pi o \delta o ́ \tau \omega ~ \pi \rho \alpha ́ \sigma \varepsilon \imath)$ kaufen.

Um diese letzte Möglichkeit zu beweisen, d.h. dass keine Wandelung gegen „simpliciter et ita, ne restitutio fiat" platzt greife, zitiert der Stephanus eben die Stelle D. 21, 1, 48, 8, was deutlich macht, wie er sie interpretierte: die simplaria venditio des Pomponius wäre der Kauf mit vertraglichem Ausschluss der Wandelung ( $\alpha \dot{v} \alpha \pi \circ \delta o ́ \tau \omega ~ \pi \rho \alpha ́ \sigma \varepsilon \imath)$. Diese Interpretation könnte doch eine Vereinfachung sein, die von der oben vorgeschlagenen Verwandlung der Bedeutung von „simplaria venditio“ beeinflusst worden war. Dass es

$28 \quad$ Lateinische Übersetzung von Zachariae von Lingenthal (Unterstreichung hinzugefügt).

29 „Doch sei es so verstanden, nur wenn der Verkäufer den Sklaven nicht einfach und so, dass die Sache nicht zurückgegeben werde, verkauft hat. Denn gegen ihn, der einfach und so, dass die Sache nicht zurückgegeben werde, verkauft hat, greift die ädilizische [Klage] keinen Platz, wie Papinianus (sic) im 48. Dig. desselben Buches und Titels [D. 21, 1, 48, 8] sagt“. 
eine Vereinfachung wäre, wäre angesichts des didaktischen Charakters des Scholions gar nicht zu verwundern, zumal es höchstwahrscheinlich schon eine krasse Vereinfachung in den ersten zwei Teilen gemacht wurde (JAKAB, 2009). Dass es das Ergebnis der erwähnten Bedeutungsverwandlung wäre, wäre mit der Tatsache kompatibel, dass auch hier eine redundante Ausdrucksform zu erkennen ist: $\alpha \pi \lambda \tilde{\omega} \zeta \kappa \alpha i ̀ ~ \alpha \nu \alpha \pi o \delta o ́ \tau \omega \pi \rho \alpha ́ \sigma \varepsilon 1$, „einfach“ und „so, dass es keine Wandelung gemacht werde“. Wiederum könnte das eine Spur des Verwandlungsvorgangs sein, indem eine nicht mehr existierende Unterscheidung zwischen „einfach“ und „Ausschluss der Wandelung“ sprachlich weiterbesteht. Ob hier eine falsche Interpretation der Konstitution C. 4, 58, 1 eine Rolle gespielt hat, kann man nicht sagen. Aber die Verwendung des Adverbs ,simpliciter“ ( $\dot{\alpha} \pi \hat{\omega} \tilde{\zeta})$ könnte ein Zeichen eines Zusammenhangs sein.

Übrigens soll man nur merken, dass sowohl beim Scholion als auch beim SRR jene zwei Meinungen bezüglich der simplaria venditio bei Pomponius, die sie entweder als Kauf geringwertiger Sachen oder als Kauf ohne Zwang des Abschlusses der stipulatio duplae verstehen wollen, überhaupt keinen Sinn haben. Hier wie da kommt keine besondere Kaufsache in Frage (es handelt sich um Sklaven), die der Voraussetzungen der erwähnten Meinungen entspräche.

4. Notiz zu den lateinischen Urkunden vom Kauf unter Abschluss der stipulatio simplae

Die simplaria venditio wird nie in den uns überlieferten lateinischen Urkunden erwähnt, doch gibt es zwei Beispiele davon, was nach der oben vorgeschlagenen Auslegung von D. 21, 1, 48, 8 für so einen Kauf gehalten werden könnte, d.h. für einen Kauf unter Abschluss der stipulatio simplae für Rechtsmängelhaftung. Die Urkunden sind TH 61 (Pompeji, 63 n. Chr.) und FIRA III 132 (Seleukia, 166 n. Chr.). Mit diesen Urkunden lässt sich kein sicherer Schluss über die simplaria venditio ziehen, aber es ist zumindest wichtig zu merken, dass beide doch kompatibel mit der vorgeschlagenen Auslegung von D. 21, 1, 48, 8 sind.

Die Urkunde der Tabulae Herculanenses lautet so (Tab. II, S. 1):

1 [hunc hominem sa]num furtis noxisque solutum esse

2 [praestari et, si qui]s eum hominem partemve eius evicerit, quo

3 [minus L. Comi]nium Primum heredemve eius habere

4 [uti frui] possidere recte liceat, simplam pecuniam r[ect]e

5 [dari, haec,] ita uti adsolet, recte praestari stipu[latus]

6 [est L. Comin]ius Primus, spopondit P. Cornelius Popp[a]eu[s]

7 [Erastus]. 
Was übersetzt hieße:

1 [Um dafür, dass dieser Mann ges]und, kein Dieb und frei von noxaler Haftung ist, 2 [zu haften, und um dann, wenn jemand diesen Mann bzw. einen Teil von ihm evinziert haben wird,

3 [sodass L. Comi]nium Primum bzw. seine Erben das Haben,

4 [das Gebrauchen und das Nutzen] nicht gewährleistet werden, das einfache Geld, 5-7 [zu geben - das alles, wie es gepflegt wird, hat P. Cornelius Poppaeus Erastus L. Cominius Primus verheißen zu gewährleisten.

Die simpla pecunia wird verheißen für den Fall der Eviktion, was nach der vorgeschlagenen Auslegung von D. 21, 1, 48, 8 eine simplaria venditio gestalten würde. Allerdings fällt es sofort auf, dass gerade auf der ersten Zeile die Sachmängelhaftung (sanum furtis noxisque solutum esse) vereinbart wird - was die vorgeschlagene Auslegung der Pomponiusstelle eindeutig zu widersprechen scheint. Sieht man aber das Datum (63 n. Chr.) der Urkunde, merkt man: Sie ist ungefähr 70 Jahre vor der Verfassung des 23. Buches ad Sabinum durch Pomponius entstanden. Das, was Pomponius wahrnehmen konnte, war nicht notwendig schon im vorigen Jahrhundert zu beobachten, oder zumindest nicht auf eine identische Weise. Außerdem hat Pomponius geschrieben (nach der vorgeschlagenen Auslegung), die Wandelung sei ausgeschlossen, wenn die simpla statt dupla für Rechtsmängelhaftung vereinbart werde. Auf der Tafel gibt es doch Sachmängelhaftung (und zwar auf Wandelung, darf man annehmen), aber nur eine geminderte Haftung, die vom Standard, der z.B. im Ädilenedikt auftaucht, abweicht: es wird versprochen, lediglich dass der Sklave gesund und kein Dieb ist, und dass keine noxale Haftung vorliegt, aber nicht dass er kein Ausreißer ist.

Die Tafel ist also doch mit der vorgeschlagenen Auslegung von D. 21, 1, 48, 8 kompatibel, da ihre Entstehung ungefähr vor 70 Jahre vor der analysierten Digestenstelle zu datieren ist, und eine Änderung der Rechtslage mittlerweile passiert sein könnte; und da in ihr doch eine Minderung der Sachmängelhaftung doch zu finden ist, wenn mit dem Ediktstandard verglichen wird; und Pomponius den Ausdruck ,in usu est " gebraucht, was nicht ausschließt, dass Gegenfälle vorliegen können, und wenn dieser Ausdruck so verstanden wird, wie Lycarion bei P. Cair. Preis. $1 \mathrm{ihn} \mathrm{zu}$ verwenden scheint (also: als Gerichtspraxis), ist bei einer simplaria venditio (Abschluss der stipulatio simplae) die Wandelung nur dann ausgeschlossen, wenn die Parteien nichts über die Sachmängel explizit vereinbart haben — was die Möglichkeit nicht ausschließt, dass sie im einzelnen Fall diese Haftung doch vereinbaren. 
Und die Urkunde FIRA III 132 lautet so:

[...] Eum puerum sanum esse ex edicto, et si quis eum puerum partemve quam eius evicerit, simplam pecuniam sine denuntiatione recte dari stipulatus est Fabullius Macer, spopondit Q. Iulius Priscus: id fide sua et auctoritate esse iussit C. Iulius Antiochus [...] et tradidisse ei mancipium s(upra) s(criptum) Eutychen bonis condicionibus. [...]

Was folgenderweise übersetzt werden könnte:

[...] Um dafür, dass der Jung gesund gemäß dem Edikt ist, zu haften, und wenn jemand ihn oder einen Teil von ihm evinziert haben wird, hat Q. Iulius Priscus Fabullius Macer verheißen, das einfache Geld ohne denuntiatio zu geben; dass es nach seiner Treue und auctoritate (=die auctoritasHaftung annehmend) so ist, hat C. Iulius Antiochus geheißen. [...] und dass er ihm den o. g. Sklaven Eutychen unter guten Bedingungen übergeben hat. [...]

Wiederum wird die stipulatio simplae für den Fall der Eviktion vereinbart, und die Wandelung wird nicht ohne Weiteres ausgeschlossen: der Verkäufer haftet dafür, dass der Sklave ,gesund gemäß dem Edikt ${ }^{30 ، ~}$ ist. Allerdings ist es auffallend, dass die Sachmängelhaftung nicht dem Standard entspricht: dass der Sklave kein Ausreißer oder Dieb ist, und dass keine noxale Haftung vorliegt, wird nicht versprochen. Die Urkunde liegt zeitlich schon nach Pomponius, weshalb eine Änderung der Praxis diesmal nicht vorstellbar ist, wie bei TH 61. Aber es spricht für die Kompatibilität der Urkunde mit der aufgestellten These über die simplaria venditio, dass es bei FIRA III 132 doch eine Minderung der Sachmängelhaftung gibt, und dass die vorgeschlagene Auslegung der Pomponiusstelle kompatibel mit Einzelfällen ist, in denen die Wandelung nicht überhaupt, sondern begrenzt ausgeschlossen wurde. Nochmal wäre es ein Fall gewesen, bei dem über die Sachmängelhaftung nicht geschwiegen worden wäre (vielmehr ist es bonis condicionibus vereinbart worden), weshalb der von Pomponius erwähnte usus, als Gerichtspraxis verstanden, sowieso da keine Anwendung finden würde.

Und dass es doch eine Abweichung vom Standard der Sachmängelhaftung in diesen Urkunden zu beobachten ist, zeigt der Vergleich mit zwei ähnlichen Urkunden, bei denen die dupla versprochen wird, und die Liste der Sachmängel dementsprechend ausführlich aufgeschrieben ist: TH 60 (Pompeji, vor 63 n. Chr.) — sanam ess[e furtis] noxaque solutam, [fugi]tivam, [erro]nnem non esse praestari [e]t [dupla]m pecuniam ex formula edicti [aedili]um, ita uti adsolet, [...] stipulata est Calatoria - und FIRA III 88

$30 \quad$ Es wäre zu hinterfragen, ob es sich um den Ädilenedikt handelt, der nicht in Seleukia galt, dessen Text aber doch möglicherweise als Maßstab außerhalb Rom gebraucht worden sein könnte. 
(Dakia, 142 n. Chr.) - sanum traditum esse, furtis noxaque solutum, erronem, fugitivum, caducum non esse praestari, et si quis eum puerum q(uo) d(e) a(gitur) partenve quam quis ex eo evicerit, q(uo) m(inus) emptorem s(upra) s(criptum) eunve ad q(uem) ea res pertinebit, uti frui habere possidereque recte liceat, tunc quantum id erit, quod ita ex eo evinctum fuerit, t(antam) p(ecuniam) duplam p(robam) r(ecte) d(ari).

III. Sprachliche Überlegungen zu D. 21, 1, 48, 8

Als letztes sind einige sprachlichen Überlegungen relevant, die zusammen mit dem Ergebnis der Analyse der Quellen die am Anfang vorgeschlagene Auslegungsmöglichkeit zu D. 21, 1, 48, 8 unterstützen können. Zu erörtern sind die Ausdrücke ,simplariarum (venditionum)“ und ,,in usu est“, über die schon einiges gesagt worden ist, die aber noch einer letzten Überlegung bedürfen.

\section{1. „Simplariarum“}

Wie gesagt, das Wort ist ein Hapaxlegomenon: die Stelle des Pomponius ist der einzige Text der gesamten lateinischen Überlieferung, in dem es auftaucht. Diese Tatsache führt sofort zum Gedanke, es wäre einfach absurd, dass hier genau dasselbe wie mit dem Adjektiv „simplex“ gemeint wäre. Dass ein anderes Wort verwendet worden ist, und zwar absolut einmalig, scheint ein sehr starker Hinweis dafür zu sein, dass etwas anders gemeint war. Die Wahl eines sehr spezifischen Wortes ohne einen besonderen semantischen Zweck ist schon bedenklich. Die Wahl eines Hapaxlegomenons ohne so einen Zweck ist völlig unvorstellbar.

Um den möglichen durch Pomponius gemeinten Sinn festzustellen, kann ein Blick auf die Struktur des Wortes nützlich sein. Der Suffix ,-ari“ (wie bei „simplaria“) weist häufig auf Zugehörigkeit, Derivation oder Zusammengehörigkeit hin, wie bei „militaris“ aus „,miles“, „palmaris“ aus ,palma“ und „calcaris“ aus „calx“. Bekannt (obwohl im späten Latein) ist jedenfalls das Adjektiv ,simplaris“", das z.B. bei Vegetius (armaturae... simplares, qui singulas [consecuntur annonas]) (11 $^{31}$ und bei Modesto (torquarii duplares, torquarii simplares) ${ }^{32}$ auftaucht. Da heißt es: „mit der singula annona verbunden“. Die Parallel zwischen ihm und ,simplaria“ bei Pomponius wurde schon von Gillaume Budé (1543, p. 350) bemerkt, und er wollte die Stelle D. 21, 1, 48, 8 eben damit erklären: simplaria wäre aus ,simpla (pecunia)“ gestaltet worden, im Sinne der stipulatio simplae, genauso wie die von ihm erwähnten actiones simplariae, duplariae und quadruplariae,

\footnotetext{
31 De re militari 2,7 .

32 De vocabulis rei militaris 6,19 .
} 
je nachdem sie in simplum, duplum oder quadruplum erteilt wurden. Auch wenn die Meinung Budés zur Bedeutung der simplariae venditiones nicht im Ganzen zu vertreten ist, denn sie geht in der Richtung der Abwesenheit des Garantiezwanges wegen der Art der Kaufsache, ist der klare von ihm unterstrichene Zusammenhang von „simplaria“ und „simpla“ zu bejahen. Die venditio simplaria ist der Kauf, der mit der „simpla“, d.h. simpla pecunia (Haftung aus der stipulatio simplae für Rechtsmängelhaftung), zusammengehört.

Und obgleich es wohl vorstellbar ist, Pomponius hätte das Wort eben für das 23. Buch ad Sabinum geschaffen, ist es auch möglich, dass in der Florentina ein Abschreibfehler begangen wurde. Man könnte nämlich vermuten, Pomponius hatte „simplarium venditionum..." mit dem auch woanders (auch wenn später) auftauchenden Adjektiv „simplaris“ geschrieben. Irgendwann, beim Kopieren vom Digest, hätte jemand den typischen Fehler einer duplicatio von drei Buchstaben begangen: ,simplariarium venditionum...". Bei der Herstellung einer weiteren Kopie hätte der Abschreiber das ,i“ weggelassen, denn das Wort ,simplariarium“ unmöglich ist, wogegen ,simplariarum“ ein ganz normales Genitiv Plural eines Adjektivs der 1. Klasse sein könnte - oder einfach wiederum aus Versehen. Dafür sprechen die Varianten des lateinischen Textes des Digests, die Dionysius Gothofredus in einer Anmerkung zur Littera Florentina erwähnt: ${ }^{33}$ simpularium (wie Corrasio) oder singularium (wie Haloander). Beide Varianten

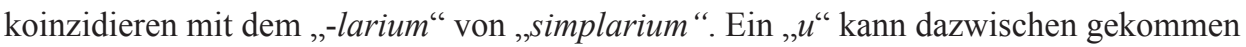
sein, und folglich eine Verwandlung von simpularium zu singularium passiert sein als Versuch, etwas weniger unwahrscheinlich zu finden.

Der griechische Ausdruck , $\dot{\alpha} \pi \lambda \tilde{\eta} \pi \rho \tilde{\alpha} \sigma ı \varsigma^{\prime \prime}$ (wie er in Bas. 19, 10, 48 auftaucht) wäre direkt aus „, $\dot{\alpha} \lambda \tilde{\eta} \chi \chi \tilde{\eta} \mu \alpha^{\prime \prime}$ geformt worden, ohne die Hinzufügung eines Suffixes wie auf Latein. Da das Adjektiv „, $\dot{\pi} \lambda \hat{\eta} \tilde{\eta}^{\prime \prime}$ nicht so spezifisch wie ,simplaria“ ist, da es auch „einfach“ bedeuten kann, ohne die „Zusammengehörigkeit mit etwas Einfachem“ auszudrücken, könnte es ein der Faktoren gewesen sein, das zur Verwirrung der Spätantiken (mit dem Ausschluss der Wandelung als Nebenwirkung und Wesenheit der simplaria venditio) geführt hat.

2. „In usu est““

Die auffallende Aussage Pomponius, „es sei üblich“, dass die Wandelung bei einfachen (oder „einfachigen“?) Käufen keinen Platz greift, ist als letztes zu besprechen. Die Ausdrucksform ,in usu“ oder Äquivalentes taucht in manchen Stellen des Digests auf, und bedeutet meistens, dass etwas in der Praxis tatsächlich gebraucht wird, wie eine Klage (D. 10, 4, 1 Ulp.) oder eine Strafe (D. 48, 15, 7 Herm.; D. 48, 1, 8 Paul.). Aber in D. 21,

33 Anm. 1 in G. Vignali (1857, p. 450). Vgl. auch C. F. Glück (1819, p. 45-46). 
1, 48, 8 wird eine Tatsache, und nicht ein Ding, als üblich bezeichnet: in usu est... ne sit. Es ist üblich, dass..., ,keinen Platz greift“. Pomponius bezieht sich auf die Tatsache, dass die Wandelung keinen Platz greift. Er sagt nämlich nicht, dass die simplariae venditiones üblich sind. Üblich ist was passiert, wenn eine von ihnen vorliegt: keine redhibitio. Das kann zwei konkreten Bedeutungen haben: dass bei solchen Käufen die redhibitio von den Parteien meistens ausgeschlossen wird (was nicht dasselbe ist, als zu sagen, solche Käufe sind die Käufe, bei denen die Wandelung ausgeschlossen wird), oder dass man bei solchen Käufen aus Gewohnheit davon ausgeht, dass die Wandelung ausgeschlossen wurde (falls sie nicht ausdrücklich vereinbart worden war). Die erste Möglichkeit ist eine reine faktische Tatsache bezüglich der Praxis auf dem Markt, die zweite ist eine juristische Aussage, die etwas wie eine Vermutung (im technischen Sinne) oder eine ergänzende Vertragsauslegung darstellt. Was genau Pomponius gemeint hat, lässt sich nicht aus dem Wortlaut entnehmen. Aber mithilfe der Analyse der Quellen könnte man doch die Hypothese aufstellen, dass was am Anfang reine Praxis (möglich gemäß D. 2, 14, 31 Ulp.) ohne juristische Folgen war, allmählich zum Grund einer juristischen Vermutung bzw. einer ergänzenden Vertragsauslegung wurde, auf der sich Lycarion in seiner Argumentation bei P. Cair. Preis. 1 zu stützen scheint. Die Formulierung dieser Vermutung/ergänzende Vertragsauslegung könnte wohl von Pomponius im Buch 23 ad Sabinum, als er den Ädilenedikt kommentierte, gemacht worden sein. Und natürlich ist es logischer einem Juristen so eine Affirmation zuzuschreiben, als eine reine faktische Aussage über die Marktpraxis.

IV. Fazit

Die Stelle D. 21, 1, 48, 8 (Pomp.) lässt sich also trotz der heutigen Einstimmigkeit um die herrschende Meinung auf eine alternative Weise interpretieren. Als Pomponius ,simplariarum venditionum causa“ gesagt hat, müsste er ,aufgrund der Käufe unter Abschluss der stipulatio simplae für Rechstmängelhaftung“ gemeint haben. Das wäre besser mit dem durch ihn erheblich spezifischen verwendeten Wort vereinbar, das entweder ein Hapaxlegomenon oder das Ergebnis eines Schreibfehlers eines komplexen Wortes (also Umwandlung von ,simplarium“) ist. Das wäre auch besser mit der Tatsache vereinbar, dass Pomponius den Ausschluss der Wandlung auf die Gewohnheit zurückführt: Es ist wohl vorstellbar, dass die nachvollziehbare gängige Praxis, eine Minderung der Sachmängelhaftung bei der Minderung der Rechstmängelhaftung zugleich zu vereinbaren, später zu einer juristischen Vermutung bzw. ergänzenden Vertragsauslegung im Fall des Schweigens bezüglich Sachmängelhaftung geführt hat.

Der Sinn dieser Verbindung zwischen Rechts- und Sachmängelhaftung, die zuerst als faktische, später als juristische Praxis gegolten haben soll, könnte eben darin 
liegen, dass der Verkäufer objektive Zweifel über den allgemeinen juristischen Zustand des Sklaven hatte (ob frei, wessen Eigentum, in welchem Zustand, mit welchen verborgenen Mängeln), wenn er eine vertragsmäßige Minderung der Haftung für Eviktion vereinbaren wollte. So für Zweifel müssten eigentlich eine Folge des objektiven Unwissens des Verkäufers über die Umstände des Sklaven sein. Es war nämlich der Fall z.B. in jenen Situationen, in denen der Verkäufer den Sklaven ohne genügende Informationen rasch erworben hatte, vielleicht sogar zusammen mit vielen anderen, um dann in weniger Zeit weiterzuverkaufen. Da beide Aspekten des Unwissens über den Zustand des Sklaven (juristisch und psychologisch-physisch) häufig zusammenfallen konnten, ist es auch logisch zu denken, dass die Minderung der Rechtsmängelhaftung von der Minderung der Sachmängelhaftung begleitet wurde.

Die Spätantiken haben offensichtlich D. 21, 1, 48, 8 und der Ausdruck „ $\dot{\alpha} \pi \lambda \tilde{\eta} \omega \dot{\omega} \eta$ “ anders verstanden. Das kann aber auf einen langen Vorgang zurückzuführen sein, der in drei Jahrhunderte passiert sein kann: die Vermutung des Ausschlusses der Wandelung bei der simplaria venditio könnte so verbreitet sein, dass der Ausdruck

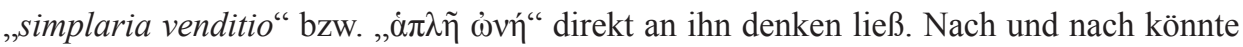
der Ausdruck aber durch Metonymie pars pro toto den Ausschluss der Wandelung eingeschlossen haben, und dann wegen der besonderen Relevanz der Sachmängelhaftung hauptsächlich deren Ausschluss bedeutet haben. So würde sie bei der Auffassung des SRR verstanden, genauso wie bei der Entstehung des Codex Justinianus (in dem C. 4, 58, 1 unter den Titel „De aedilicibus actionibus“ gestellt wurde) und bei der Auffassung des analysierten Scholions des Stephanus. Sowohl im SRR als auch im Scholion wäre eine Spur der früheren Unterscheidung in der Redundanz „einfach, mit Abschluss“ zu erkennen. Aber SB 1813173 ist ein Grund zu denken, dass in der Praxis in Ägypten die Unterscheidung zwischen , $\dot{\alpha} \pi \lambda \tilde{\eta} \dot{\omega} v \eta^{\prime \prime}$ und Ausschluss der Sachmängelhaftung weiterbestanden hat. Frühere Beispiele von Käufen unter Abschluss der stipulatio simplae ohne einen absoluten Ausschluss der Wandelung sind in TH 61 und FIRA III 132 zu finden, aber, wie erklärt, sie sind jedenfalls mit der vorgeschlagenen Auslegung von D. 21, 1, 48, 8 kompatibel.

München, Juli 2018.

\section{Literaturverzeichnis}

ACCURSIUS. Magna Glosa. Lugduni: [s.n.], 1627. v. 1.

ANKUM, Hans. Gaius en Paulus, vertaald door J. E. Spruiten K. E. M. Bongenaar. Zeitschrift der Savigny-Stiftung für Rechtsgeschichte: Romanistische Abteilung, Österreich, v. 106, n. 1, p. 626$632,1989$. 
BUDÉ, Guillaume. Annotationes Gulielmi Budaei Parisiẽsis, secretarii regii, in quatuor et viginti Pandectarum libros. Pariisis: Imprimebat Michaël Vascosanus, 1543.

D’ORS, Álvaro. El digesto de Justiniano. Pamplona: Aranzadi, 1972. v. 2.

DERNBURG, Heinrich; SOKOLOWSKI, Paul von. System des römischen rechts. Berlin: Müller, 1912.

DONADIO, Nunzia. La tutela del compratore tra actiones aediliciae e actio empti. Milano: Giuffrè, 2004.

DONELLUS, Hugo. Commentariorum iure civili, 13, 3, 5. In: Opera omnia. Florenz: $[s$. n.], 1861.

ERNST, Wolfgang. Neues zur sachmängelhaftung aufgrund des Ädilenedikts. Zeitschrift der Savigny-Stiftung für Rechtsgeschichte: Romanistische Abteilung, Österreich, v. 116, n. 1, p. 208$221,1999$.

FORCELLINI, Egidio. Totius latinitatis lexicon. Oxford: Oxford University Press, 1835. v. 4.

GEORGES, Karl Ernst. Ausführliches lateinisch-deutsches handwörterbuch. Darmstadt: Wissenschaftliche Buchgesellschaft, 1998. v. 2.

GILLIAM, James Frank. The sale of a slave through a Greek 'diploma'. The Journal of Juristic Papyrology, Warsaw, v. 16-17, p. 63-70, 1971.

GLARE, Peter G. W. (ed.). Oxford latin dictionary. Oxford: The Clarendom Press, 1945. v. 2.

GLÜCK, Christian Friedrich von. Ausführliche erläuterung der pandecten nach Hellfeld: ein kommentar. Erlangen: Palm \& Enke, 1819. v. 20, s. 1.

HONSELL, Heinrich. Von den ädilizischen rechtsbehelfen zum modernen sachmängelrecht. In: NÖRR, Dieter; SIMON, Dieter. Gedächtnisschrift für Wolfgang Kunkel. Frankfurt am Main: Vittorio Klostermann, 1984.

HONSELL, Heinrich; MAYER-MALY, Theo; SELB, Walter. Römisches recht. Berlin-Heidelberg: Springer, 1987.

HULOT, Henri. Les cinquante livres du Digeste ou des Pandectes de l'empereur Justinien. Metz: Behmer et Lamort, 1804. v. 3.

IMPALLOMENI, Giambattista. L'editto degli edili curuli. Padova: Cedam, 1955.

JAKAB, Éva. Cavere und haftung für sachmängel: zehn argumente gegen Berthold Kupisch. In: JAKAB, Éva; ERNST, Wolfgang (org.). Kaufen nach römischem recht: antikes erbe in den europäischen Kaufrechtsordnungen. Berlin: Springer, 2009.

JAKAB, Éva. Praedicere und cavere beim Marktkauf: Sachmängel im griechischen und römischen Recht. München: Beck, 1997. 
JAKAB, Éva. Prozess um eine entlaufene sklavin (P. Cair. Preis. 1): vertrag in der provinzialen rechtskultur. Zeitschrift der Savigny-Stiftung für Rechtsgeschichte: Romanistische Abteilung, Österreich, v. 135, n. 1, p. 474-526, 2018.

KASER, Max; KNÜTEL, Rolf; LOHSSE, Sebastian. Römisches privatrecht. 21. ed. München: Beck, 2017.

KUPISCH, Berthold. Römische sachmängelhaftung: ein beispiel für die 'ökonomische analyse des rechts'? Legal History Review (Tijdschrift voor Rechtsgeschiedenis), v. 70, p. 21-54, 2002.

LENEL, Otto. Palingenesia iuris civilis. Leipzig: Tauchnitz, 1889. v. 2.

LEWALD, Hans. Eine synchoresis aus der zeit des Commodus: Papyrus Rainer G. 25 817. In: Festschrift für Arangio-Ruiz, Neapel: Dott. Eugenio Jovene, 1953. v. 3.

MEMMER, Michael. Der „schöne Kauf“ des „guten Sklaven“. Zeitschrift der Savigny-Stiftung für Rechtsgeschichte: Romanistische Abteilung, Österreich, v. 107, n. 1, p. 1-45, 1990.

MEYER, Paul Martin. Neue juristische Papyrus-Urkunden und Literatur. Zeitschrift für Vergleichende Rechtswissenschaft, Stuttgart-Feuerbach, v. 39, p. 220-282, 1921.

MITTEIS, Ludwig. Grundzüge und Chrestomathie der Papyruskunde. Leipzig: B. G. Teubner, 1912.

PARTSCH, Joseph. P. Freib. II. Heidelberg: [s. n.], 1916.

PRINGSHEIM, Fritz. The greek law of sale. Weimar: H. Böhlaus, 1950.

RABEL, Ernst. Grundzüge des römischen privatrechts. 2. ed. Darmstadt: Wissenschaftliche Buchgesellschaft, 1955.

SALOMONS, Robert Paul. P. Cair. Preis. (2). Bruxelles: Association égyptologique Reine Elisabeth, 2014.

SCHIPANI, Sandro (dir.). Iustiniani Augusti Digesta seu Pandectae: testo e traduzione. Milano: Giuffrè, 2011. v. 4.

SELB, Walter; KAUFHOLD, Hubert. Das syrisch-römisches rechtsbuch. Wien: Österreichischen Akademie der Wissenschaften, 2002. v. 1.

URBANIK, Jakub. P. Cairo Masp. I 67120 recto and the liability for latent defects in the late antique slaves sales: or back to 'epaphe'. The Journal of Juristic Papyrology, Warsaw, v. 40, p. 219-247, 2010.

VIGNALI, Giovanni. Corpo del diritto: Digesto. Napoli: Pezzuti, 1857. v. 3.

WATSON, Alan. The digest of justinian. Philadelphia: University of Pennsylvania, 1985. v. 2.

WEIß, Egon. Peregrinische manzipationsakte. Zeitschrift der Savigny-Stiftung für Rechtsgeschichte: Romanistische Abteilung, Österreich, v. 37, n. 1, p. 136-176, 1916. 
WENGER, Leopold. Die quellen des römischen rechts. Wien: A. Holzhausen, 1953.

WINDSCHEID, Bernhard; KIPP, Theodor. Lehrbuch des Pandektenrechts. Frankfurt am Main: Rütten \& Loening, 1906. v. 2.

WOLFF, Hans Julius. Das recht der griechischen papyri Ägyptens in der zeit der Ptolomäer und des prinzipats. München: C.H. Beck, 2002. 
